\title{
Multimodal Characterization of Neural Networks Using Highly Transparent Electrode Arrays
}

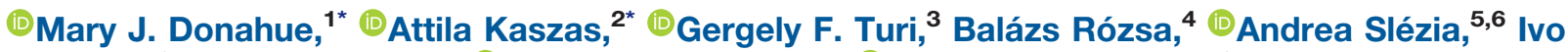
Vanzetta, ${ }^{2}$ Gergely Katona, ${ }^{7}$ Christophe Bernard, ${ }^{5}{ }^{\circledR}$ George G. Malliaras, ${ }^{8}$ and Adam Williamson ${ }^{5,6}$

https://doi.org/10.1523/ENEURO.0187-18.2018

${ }^{1}$ Department of Bioelectronics, Ecole Nationale Supérieure des Mines, Centre of Microelectronics in Provence, Gardanne 13541, France, ${ }^{2}$ Aix Marseille Univ, CNRS, INT, Inst Neurosci Timone, Marseille, France, ${ }^{3}$ Department of Psychiatry, Division of Systems Neuroscience, Columbia University and Research Foundation for Mental Hygiene, New York State Psychiatric Institute, New York, NY 10032, ${ }^{4}$ Laboratory of 3D Functional Network and Dendritic Imaging, Institute of Experimental Medicine, Hungarian Academy of Sciences, Budapest H-1083, Hungary, ${ }^{5}$ Aix Marseille Univ, INSERM, INS, Inst Neurosci Syst, Marseille, France, ${ }^{6}$ Neuroengineering Research Group, Interdisciplinary Excellence Center, Department of Medical Microbiology and Immunobiology, University of Szeged, Szeged 6720, Hungary, ${ }^{7}$ Two-Photon Measurement Technology Research Group, Pázmány Péter Catholic University, Budapest $\mathrm{H}-1083$, Hungary, and ${ }^{8}$ Electrical Engineering Division, Department of Engineering, University of Cambridge, Cambridge CB3 OFA, United Kingdom

\section{Visual Abstract}
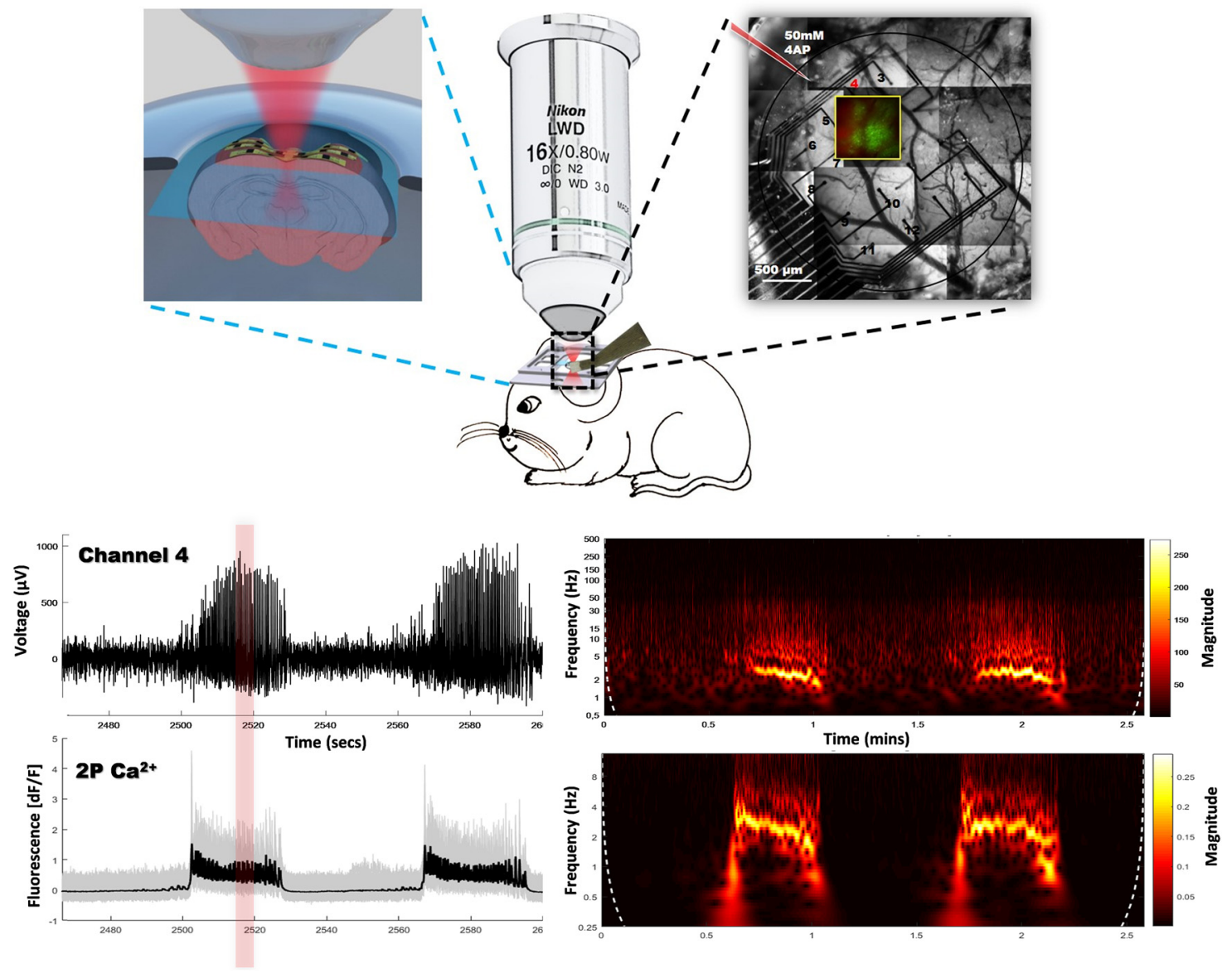


\section{Significance Statement}

Electrophysiological recordings, with varying degrees of invasiveness, are the traditional method for measuring neural activity, possessing the capability to measure both individual neurons and populations of neurons. Imaging methods, such as computed tomography scans and functional magnetic resonance imaging, have been developed to accomplish less invasive characterization of neuronal activity; traditionally offering good spatial or relatively high temporal resolution, yet resolution of individual neurons cannot be achieved. Two-photon (2P) imaging enables network-wide analysis with cellular resolution on a faster timescale with high spatial fidelity. This study presents a method for the combination of $2 \mathrm{P}$ imaging and electrophysiological recordings with highly transparent arrays of organic electrodes, presenting a powerful tool to simultaneously acquire the electrical and optical activity of neural circuits.

Transparent and flexible materials are attractive for a wide range of emerging bioelectronic applications. These include neural interfacing devices for both recording and stimulation, where low electrochemical electrode impedance is valuable. Here the conducting polymer poly(3,4-ethylenedioxythiophene):poly(styrenesulfonate) (PEDOT:PSS) is used to fabricate electrodes that are small enough to allow unencumbered optical access for imaging a large cell population with two-photon (2P) microscopy, yet provide low impedance for simultaneous high quality recordings of neural activity in vivo. To demonstrate this, pathophysiological activity was induced in the mouse cortex using 4-aminopyridine (4AP), and the resulting electrical activity was detected with the PEDOT:PSS-based probe while imaging calcium activity directly below the probe area. The induced calcium activity of the neuronal network as measured by the fluorescence change in the cells correlated well with the electrophysiological recordings from the cortical grid of PEDOT:PSS microelectrodes. Our approach provides a valuable vehicle for complementing classical high temporal resolution electrophysiological analysis with optical imaging.

Key words: electrophysiology; neuroengineering; organic electronics; PEDOT:PSS; transparent electronics; two-photon imaging

\section{Introduction}

Network-level brain functions result from the activity of large populations of neurons. Optical and electrophysiological techniques have been developed to interrogate the functions of the interconnected neurons within networks and to track the activity of as many individual neurons as possible. High-density neural probes for electrophysiological applications record hundreds of neurons simulta-

Received May 13, 2018; accepted November 20, 2018; First published December 21, 2018.

G.K. and B.R. are owners of Femtonics and the patent WO2010076579. All other authors declare no competing financial interests.

Author contributions: M.J.D., G.F.T., and A.W. designed research; M.J.D. and A.K. performed research; I.V., B.R., G.K., and A.S. contributed unpublished reagents/analytic tools; G.F.T., C.B., A.S., and G.G.M. analyzed data; M.J.D, A.K., G.F.T., I.V., A.S., C.B., and A.W. wrote the paper.

This work was supported by the European Research Council (ERC) under the European Union's Horizon 2020 Research and Innovation Programme (Grant 716867). A.K. was supported by EC Marie Curie Intra-European Fellowship (ImagINE, Grant 625372). M.J.D. was supported by the Fondation pour la Recherche Médicale Grant DBS20131128446. B.R. and K.G. were supported by ERC682426, KFI-2016-0177, GINOP-2016-00979, and NVKP-2016-0043.

${ }^{*}$ M.J.D. and A.K. contributed equally to this work.

Correspondence should be addressed to Dr. Adam Williamson, AixMarseille Université, Inserm U1106 INS, Institut de Neurosciences des Systèmes, Marseille, 13005, France. E-mail: adam.williamson@univ-amu.fr.

https://doi.org/10.1523/ENEURO.0187-18.2018

Copyright (C) 2018 Donahue et al.

This is an open-access article distributed under the terms of the Creative Commons Attribution 4.0 International license, which permits unrestricted use, distribution and reproduction in any medium provided that the original work is properly attributed. neously. This technique allows for the direct assessment of individual neuronal activity (firing of action potentials) as well as population activity of many neurons (field potentials; Buzsáki and Draguhn, 2004). The main disadvantage of electrophysiological recordings is poor spatial resolution (Taketani and Baudry, 2010). For example, implantable probe geometry does not allow the recording of all neurons belonging to one functional unit, such as a cortical column. Calcium $\left(\mathrm{Ca}^{2+}\right)$ imaging records large populations of neurons with high spatial resolution, offering an option to overcome this disadvantage (Stosiek et al., 2003). The $\mathrm{Ca}^{2+}$ signal, however, is an indirect measure of cellular activity, thus it would be ideal to combine physiologic and optical techniques. To achieve this combination, the electrophysiological signal should be measured where the optical signal is obtained. This approach requires a highly transparent electrophysiological device to allow efficient collection of the optical signal. Although recent developments for this scenario have been demonstrated in vitro (Chang et al., 2011; Benfenati et al., 2013; Scott et al., 2013), the simultaneous acquisition of neural activity in vivo has remained a technical challenge.

Studies aiming to combine in vivo single cell electrophysiological recordings with $2 \mathrm{P}$ imaging use intracellular or extracellular glass electrodes (Helmchen et al., 2001; Svoboda and Yasuda, 2006; Kitamura et al., 2008) employing a $\mathrm{Ag} / \mathrm{AgCl}$ electrode to close the recording circuit. Alternatively, a metal electrode may be chronically implanted 
in the contralateral hemisphere, while the image collection is conducted at physical distance (Malvache et al., 2016), a method which assumes high correlation between the two recording sites. These methods have limitations including use of delicate recording pipettes, limited number of recording sites and the necessity to locate the metal parts of the electrode outside the field of view as it is prone to generate noise due to the photoelectric effect (Kozai and Vazquez, 2015). Flexible materials and micro-fabrication techniques have been explored over recent years to solve the former two limitations. The photoelectric effect, however, complicates the simultaneous use of optical and electrophysiological signal recording for a great deal of materials and engineering approaches. An in vitro study by Kuzum et al. (2014) well demonstrated the possibility to image hippocampal slices using confocal and $2 \mathrm{P}$ techniques through a flexible graphene electrode array, benefitting from the high-transparency of the electrode material and interconnecting lines. The used Kapton substrate, however, somewhat hinders the overall optical transparency and the $50 \times 50 \mu \mathrm{m}$ electrode surface has high electrochemical impedance $(\sim 500 \mathrm{k} \Omega$ at $1 \mathrm{kHz})$, the main requirement for capturing high-quality electrophysiological signals (Franks et al., 2005; Williams et al., 2007). In a separate study on flexible graphene electrodes, a high level of transparency was exhibited by Park et al. (2014) using a parylene $\mathrm{C}(\mathrm{PaC})$ substrate. Fluorescence imaging through the array was possible in vivo, as well as electrophysiological signal recording and optogenetic stimulation of the underlying tissue, however, relatively large electrodes are used ( $150 \mu \mathrm{m}$ in diameter) with $1-\mathrm{kHz}$ impedance values reduced only to $\sim 250 \mathrm{k} \Omega$. Recent work has pushed toward improved technologies offering in vivo electrophysiology and optical recording capabilities. These reports include arrays of $100 \times 100 \mu \mathrm{m}$ graphene electrodes (963-k $\Omega$ average impedance) with high transparency (Thunemann et al., 2018) as well as a study by Qiang et al. (2018) using a gold nano-mesh patterning technique, creating electrodes with diameters down to 20 $\mu \mathrm{m}$ with an average $1-\mathrm{kHz}$ impedance value of $130.3 \mathrm{k} \Omega$, employing a similar material system to ours. Main differences include: insulation material (SU8 vs PaC), overall higher electrode impedance $(\sim 5 \times)$ and device thickness $(\sim 4 \times)$, but improved transparency at the mesh's conduction lines and recording sites.

In this report, we demonstrate an extremely flexible ( $\sim 4-\mu \mathrm{m}-$ thick) cortical microelectrode array $(25 \times 25 \mu \mathrm{m}$ electrode size), with moderate channel density deposited on an optically transparent $\mathrm{PaC}$ substrate. The high overall transparency in combination with low impedance values $(\sim 25 \mathrm{k} \Omega$ at $1 \mathrm{kHz})$ enables electrophysiological and optical recordings from the same location. To achieve low impedance, we employ the wellknown ionic/electronic conducting polymer poly(3,4ethylenedioxythiophene):poly(styrenesulfonate) (PEDOT: PSS). PEDOT:PSS is applied at the electrode sites, efficiently reducing the impedance and allowing for minimization of the contact lines and electrode area. With this approach, unobstructed imaging may be performed through $>95 \%$ of the substrate area intended for optical and electrical measurement. The micron-scale organic electrode grid maximizes the overall optical transparency and allows for simultaneous $2 \mathrm{P}$ imaging of the underlying tissue.

\section{Materials and Methods}

\section{Device fabrication}

The organic electrode grid fabrication process is based on previously reported methods (Khodagholy et al., 2013, Sessolo et al., 2013). An initial PaC film was deposited on glass slides using a SCS Labcoater 2 with a resulting thickness of $2 \mu \mathrm{m}$. Once the subsequent fabrication steps are completed, this $\mathrm{PaC}$ layer is delaminated from the glass, thus acting as the final substrate and providing the flexibility for the Electrocorticography (ECoG) array. Photolithography and lift-off processes were employed to pattern metal interconnects on top of the PaC substrate. This was performed using a positive photoresist (Shipley MICROPOSITTM S1813), a SUSS MJB4 UV broadband contact aligner and MF26 developer. A 2-nm chromium adhesion promoting layer and $100 \mathrm{~nm}$ of gold were thermally evaporated onto the substrates and the samples were immersed in an acetone bath to define the interconnects through lift-off. To electrically insulate the metal lines, a second $\mathrm{PaC}$ film was deposited on the devices to thicknesses of $2 \mu \mathrm{m}$, using the same deposition process as before, however with 3-(trimethoxysilyl)propylmethacrylate present in the chamber (by addition of a droplet to the chamber wall) to act as an adhesion promoter. Subsequently, a dilute solution of Micro-90 industrial cleaner was spin coated onto this insulation layer, followed by the deposition of a sacrificial $\mathrm{PaC}$ layer $(2 \mu \mathrm{m})$. These steps allow the sacrificial layer to later be peeled off from the substrate, defining PEDOT:PSS at the electrode recording sites. The $\mathrm{PaC}$ layers were etched by reactive ion etching (RIE) in an Oxford 80 Plasmalab plus with an $\mathrm{O}_{2} / \mathrm{CHF}_{3}$ plasma to open the electrode sites by creating an opening down to the gold interconnect. AZ9260 photoresist was used as an etch mask, patterned using the UV contact aligner and AZ developer. A dispersion of PEDOT:PSS (CleviosTM PH 1000 from Heraeus Holding $\mathrm{GmbH}$ ), 5 volume $\%$ ethylene glycol, 0.1 volume \% dodecyl benzene sulfonic acid, and $1 \mathrm{wt} \%$ of (3-glycidyloxypropyl)trimethoxysilane was spin coated onto the substrates to attain a thickness of $200 \mathrm{~nm}$. The sacrificial PaC layer was peeled off removing superfluous PEDOT:PSS and defining the electrode sites. The devices were baked for $1 \mathrm{~h}$ at $140^{\circ} \mathrm{C}$ to crosslink the film. Finally, the devices were immersed in deionized water (DIW) to remove low molecular weight compounds. This DIW soak also facilitates the delamination of the final flexible electrode array from the glass slide.

\section{Electrophysiological signal acquisition and processing}

Neural data were recorded using a RHD2132 Intan technology amplifier board. Zero insertion force clips (ZIFClip) were used to connect directly to the ECoG devices, along with an adaptor chip to connect to the Intan technology head stage. A sampling rate of $20 \mathrm{kHz}$ was used and data were stored in 16-bit format. Data analysis was 
A

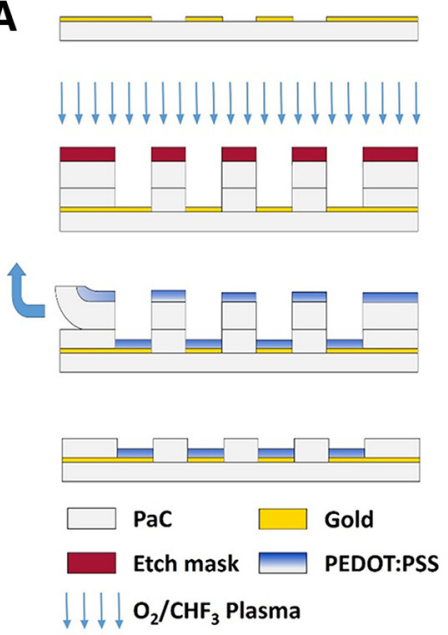

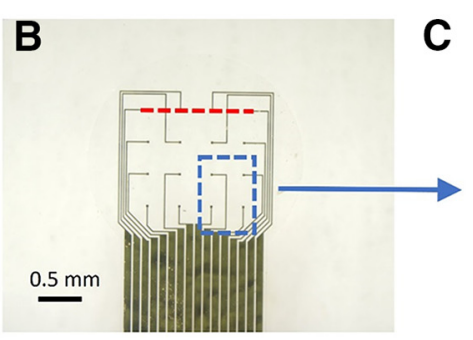
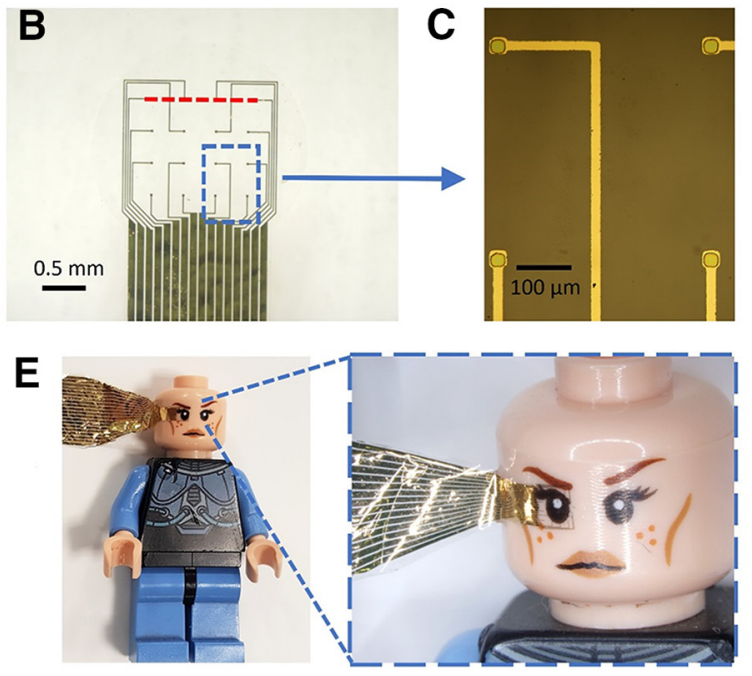

D

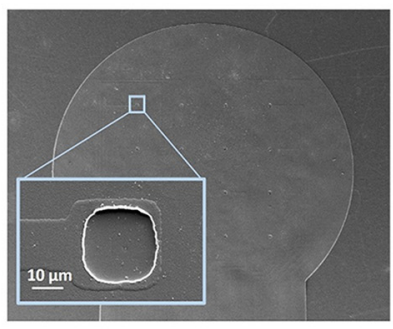

$\mathbf{F}$

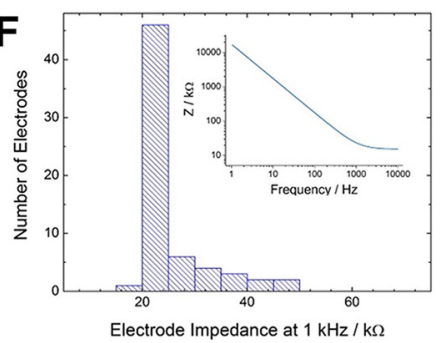

Figure 1. Organic cortical electrode grid. $\boldsymbol{A}$, Cross-sectional schematic of the microfabrication process corresponding to the area in $\boldsymbol{B}$ indicated by the red dotted line. Note: illustrative view is not to scale $\boldsymbol{B}$, Top view of the electrode array. $\boldsymbol{C}$, Microscopic image of individual organic PEDOT:PSS electrode sites in the area of the array indicated by the blue square in $\boldsymbol{B}$. $\boldsymbol{D}$, Scanning electron microscope image of the full ECoG array with a zoomed-in view of one recording site in the inset. $\boldsymbol{E}$, Eye of a Lego toy clearly visible through the device (transparent area intended for imaging centered on the eye; note the bundle of contact lines visible extending to the left of the toy). $\boldsymbol{F}$, Histogram of 64 electrode impedances from four devices at $1 \mathrm{kHz}$ with an example electrochemical impedance spectrum in the inset.

conducted using MATLAB (MathWorks). A $400 \mathrm{~Hz}$ low pass filter was applied to the acquired signal.

\section{Surgical procedure}

Transgenic Thy1-GCaMP6f mice (Dana et al., 2014) with neurons expressing a fluorescent calcium indicator (14-16 weeks old, three males) were housed according to institutional regulations in the animal facilities of corresponding universities. On the day of surgery, animals were sedated with $2 \%$ isoflurane, then fixed in a stereotactic frame and kept under $4 \%$ sevoflurane anesthesia. After a subcutaneous lidocaine injection, the skull was exposed and a cranial window (4 $\mathrm{mm}$ in diameter) was drilled above the somatosensory cortex. A head plate was mounted onto the skull by dental acrylic, and the animal was placed into the microscope setup using the head plate. The temperature of the mouse was monitored and controlled using a rectal probe and heated mouse pad set to $38^{\circ} \mathrm{C}$. During the recording procedure the anesthesia was maintained with a $1.2 \%$ isoflurane and oxygen mixture. The surface probe was positioned onto the craniotomy and covered with artificial cerebrospinal fluid, containing: $124 \mathrm{mM} \mathrm{NaCl}, 26 \mathrm{mM} \mathrm{NaHCO}_{3}, 3.5 \mathrm{mM}$ $\mathrm{KCl}, 1 \mathrm{mM} \mathrm{MgCl} 2,1 \mathrm{mM} \mathrm{CaCl}_{2}$, and $10 \mathrm{mM}$ D-glucose. A coverslip was gently placed on top of the surface probe, and a tile map was acquired by an infrared CCD camera through a $4 \times$ objective. 4-Aminopyridine (4AP) was used to evoke seizure-like activity in the cortex. The drug was pressure injected via a glass pipette containing $50 \mathrm{mM}$ 4AP with $20 \mu \mathrm{M}$ Alexa Fluor 594 as a red dye for marking the injection site. The drug injection was positioned underneath both the cover glass and the probe $\sim 500 \mu \mathrm{m}$ away from the imaging field of view, at a depth of $500 \mu \mathrm{m}$, using a micromanipulator (PatchStar, Scientifica). All pro- cedures were approved by the Aix-Marseille Université ethical committee and were in accordance with guidelines of the corresponding institutes.

\section{P imaging}

At the beginning of the experiments, a reference $z$-stack of the volume was acquired on a dual-scanhead 2P microscope (FemtoS-Dual, Femtonics Ltd) equipped with a femtosecond pulsed laser tuned to $920 \mathrm{~nm}$ (Mai Tai HP, SpectraPhysics) using a Nikon LWD 16x/0.8 NA objective. A single acquisition plane was selected and fullframe imaging was started in resonant scanning mode at $30.9375 \mathrm{~Hz}$. The control of imaging and the trigger of electrophysiological recordings was done using the microscope's acquisition software (MESc). We used the MESc and the MES software packages (Femtonics Ltd) to analyze imaging data (Katona et al., 2012).

\section{Results}

The developed organic electrode device, produced through the microfabrication steps described in the Materials and Methods section, is presented in Figure 1. Figure $1 A$ shows the schematic outline of the fabrication process. The dashed line in Figure $1 B$ corresponds to the cross-sectional area shown in Figure $1 A$. Optical images of the resulting grid of organic electrodes are given in Figure 1B,C. Overall, the micro-electrode grid consists of gold lines and 16 PEDOT:PSS recording sites patterned on a flexible $\mathrm{PaC}$ substrate. The interconnects are insulated using a secondary layer of $\mathrm{PaC}$, resulting in a thin $(\sim 4 \mu \mathrm{m})$ overall final device, extremely conformable to the surface of the brain. Openings etched in a third sacrificial layer of $\mathrm{PaC}$ (and through the insulation layer) are used to selectively pattern PEDOT:PSS to the desired electrode 


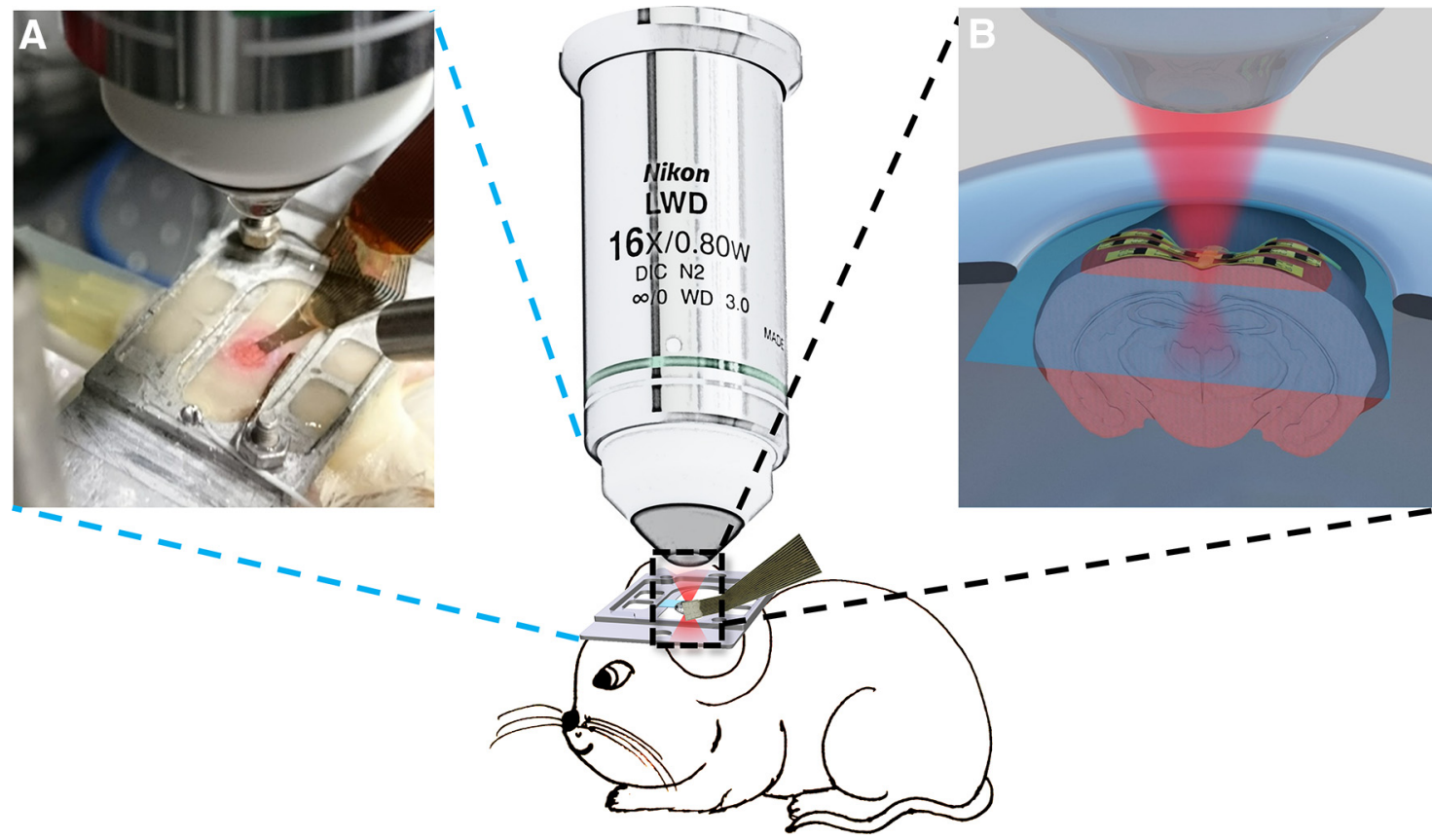

Figure 2. Layout of the recording setup with $2 \mathrm{P}$-compatible electrode array. Head-fixed, anesthetized animal under the $2 \mathrm{P}$ microscope with the grid of organic electrodes in place, monitoring electrophysiological activity of the cortex. $\boldsymbol{A}$, Photo of anesthetized animal's headplate with the probe positioned on the cortex under the objective. $\boldsymbol{B}$, The infrared laser beam (pseudo-color red) can pass through the grid of organic electrodes, as well as the subsequent visible emitted fluorescence, measured at a depth of up to $1 \mathrm{~mm}$ into the tissue, monitoring activity across the entire scanned plane (blue). Cortical electrophysiological recordings are then compared to the calcium signal measured by the $2 \mathrm{P}$ system for dual characterization of the neural network activity.

sites through a previously developed peel-off method (Khodagholy et al., 2013; Sessolo et al., 2013). Figure $1 B$ shows the resulting 16-electrode array patterned on a 2.5-mm diameter PaC substrate area. Each electrode recording site is $25 \times 25 \mu \mathrm{m}$, with an interelectrode spacing of $400 \mu \mathrm{m}$. A close-up view of a representative area indicated in blue is shown in Figure $1 C$, with the conducting polymer coating visible on four electrode sites. A scanning electrode microscope (SEM) image of the entire eletrode array is shown in Figure 1D, with a zoomed-in view of one electrode in the inset. Utilization of PEDOT:PSS at the recording sites is integral for this probe, making it possible to maintain low electrochemical electrode impedance. The $1-\mathrm{kHz}$ impedance values of 64 electrodes from four devices are shown in Figure $1 F$, all below $50 \mathrm{k} \Omega$ with an average impedance of $25.8 \mathrm{k} \Omega$; a value very well suited for electrophysiological recordings. The possibility to attain this impedance range with electrodes and interconnects on the tens of micrometers range allows for maximizing the area of transparent substrate which can be used for imaging. The transparency facilitates straightforward alignment and orientation of the array with biological structures below the grid. Figure $1 E$ demonstrates these features, with the electrode grid placed on one eye of a Lego toy figure.

As seen in Figure 2, the implemented grid of electrodes with 16 recording sites is placed on the surface of the cortex, directly in the path of the $2 \mathrm{P}$ scan. This provides multiple sites for electrophysiological measurements during $2 \mathrm{P}$ calcium imaging, representing a significant advantage over a single electrophysiological recording site of a glass pipette. Figure $2 A, B$ shows the layout of the experimental setup. The anesthetized mouse was head-fixed in the $2 \mathrm{P}$ setup and the grid of organic electrodes was placed over the visual cortex. The flexibility of the organic electrodes and the substrate allows for continuous contact with the brain surface, ensuring a good electrochemical interface between the tissue and the probe. The surface probe was covered with a glass cranial window carefully aligned to leave ample space for an entry point of a glass injection pipette used to deliver 4-AP (50 mM), a well-known method to induce epileptiform activity (Voskuyl and Albus, 1985; Szente and Baranyi, 1987; Avoli et al., 2002). During 2P imaging sessions, the emitted green fluorescence of the neuronal calcium indicator under the flexible grid was recorded simultaneously with the electrophysiological activity (Fig. 3).

The orientation of the flexible grid of organic electrodes on the surface of the cortex is shown in Figure $3 A$. The inset of Figure $3 A$ and zoomed view in Figure $3 B$ show the average of 60 frames from a $2 \mathrm{P}$ time sequence measurement, imaged through the electrode grid $200 \mu \mathrm{m}$ below the cortical surface. Neurons here expressed GCaMP6f (green) as a calcium indicator, while Alexa Fluor 594 (red) labeling as a fluorescent marker was used to follow the spread of the 4-AP solution after injection. While Figure $3 A$ numbers all available electrode sites used for recording, the numbering indicated in Figure $3 B$ shows those electrodes that were located in the 2P field-of-view. Electrophysiological local field potential (LFP) recordings for nearby electrodes are shown in Figure $3 C$, with a zoomed view of electrode 4 in Figure $3 D$, as this electrode is the 
A

D

$\mathbf{F}$
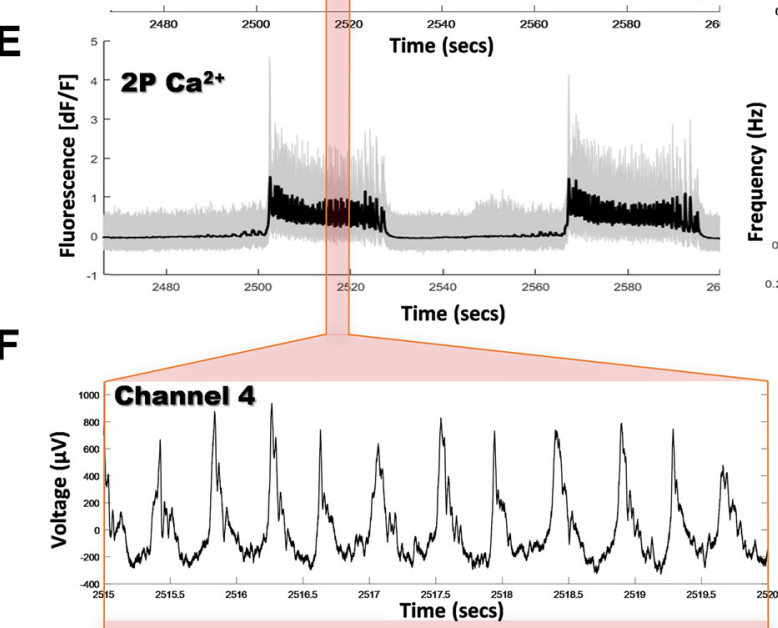

G

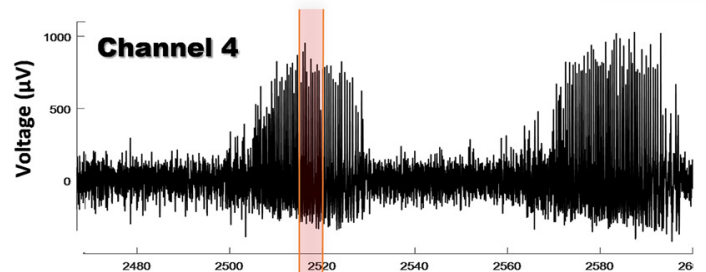

B

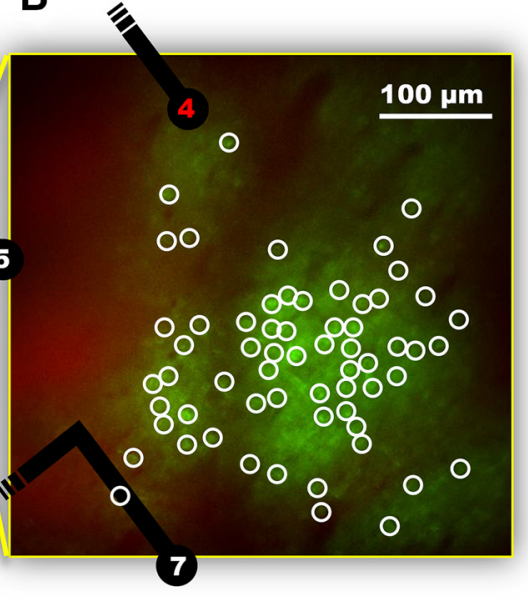

C

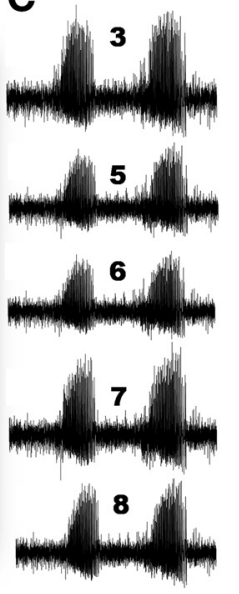

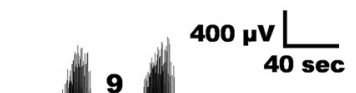

40 sec
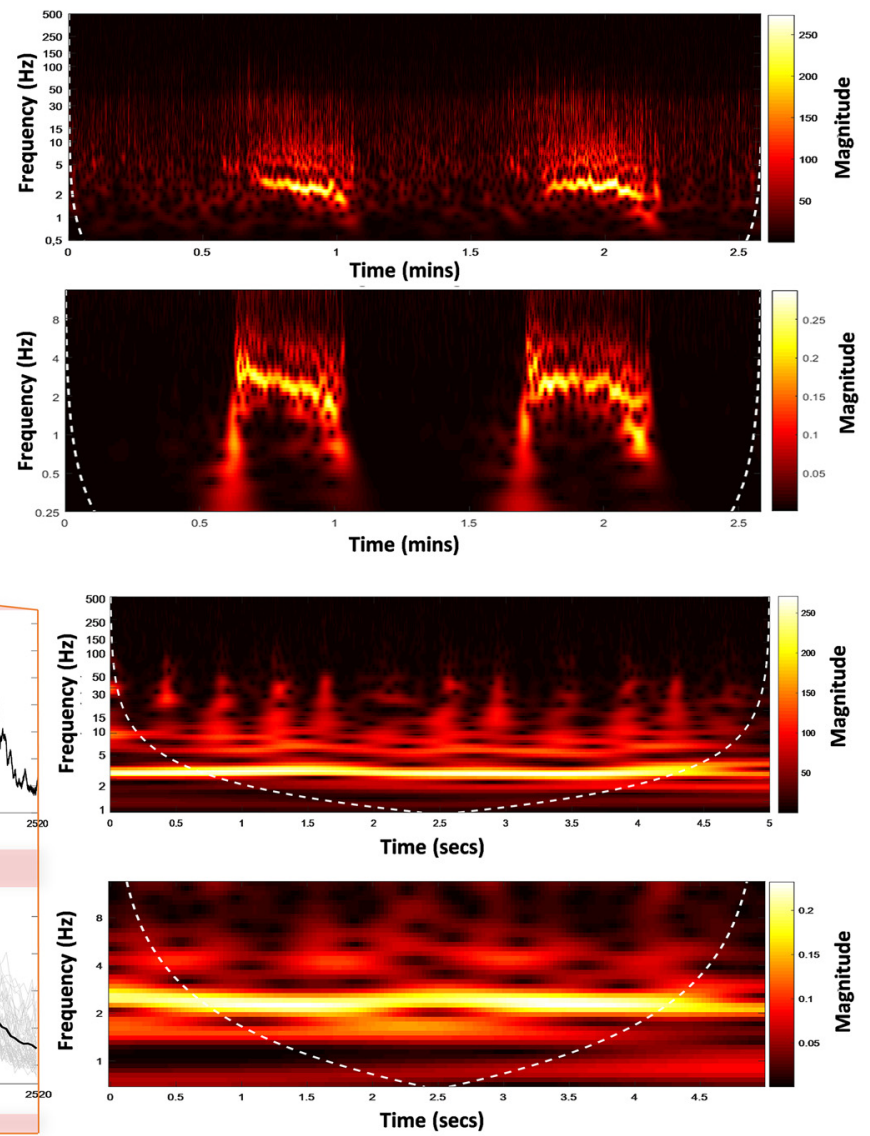

Figure 3. Simultaneous in vivo 2P imaging and cortical electrophysiological recording. $\boldsymbol{A}$, Infrared camera picture montage of the flexible 16-channel cortical electrode array positioned in a craniotomy above the primary visual cortex of a Thy1-GCaMP6f mouse. B, Average image of 60 frames from a 2P time sequence measurement of GCaMP6f (green) and Alexa Fluor 594 (red) labeling. The selected cells $(n=63$, white circles) were located $200 \mu \mathrm{m}$ below the cortical surface. Electrode locations in this area are indicated. $\boldsymbol{C}$, LFP recordings from channels depicted in $\boldsymbol{A}$. $\boldsymbol{D}, \boldsymbol{E}$, Simultaneous LFP recording (D, left) and calcium imaging (E, left) from single cells (gray) and the average (black) of interictal and ictal periods evoked by $50 \mathrm{mM} 4-\mathrm{AP}$ injection. Right panels, Corresponding continuous 1-D wavelet transform analysis. $\boldsymbol{F}, \boldsymbol{G}$, Zoomed-in view of ictal events on LFP (F, left) and calcium traces ( $G$, left) for single cells ( $G$, gray) or the population average (G, black). Right panels, Corresponding continuous $1-\mathrm{D}$ wavelet transform analysis.

most relevant regarding spatial proximity. The data represent an example of seizure activity within the tissue induced by an injection of $50 \mathrm{mM} 4-\mathrm{AP}$ (injection pipette shown in Fig. $3 A$ ). The LFP recording of electrode 4 is compared with $2 \mathrm{P}$ calcium imaging data in Figure $3 D-G$. Fluorescence changes, arising from fluctuations in intracellular calcium concentrations (Fig. $3 E$, left), clearly correspond to the simultaneously recorded electrophysi- 
ological fluctuations (Fig. 3D, left). Calcium imaging data are shown for single cells (gray, $n=63$ ) as well as the average of the population response (black). Figure $3 D, E$, right panels, shows the corresponding continuous 1-D wavelet transform analysis, for the frequency analysis of the average responses. Figure $3 F, G$ presents a zoomed-in view of the area indicated in red in Figure $3 D, E$. On this timescale ictal events on LFP (Fig. 3F) and calcium traces (Fig. 3G) for both single cells (gray traces) and the population average (black trace) are also very well correlated. These measurements demonstrate the simultaneous monitoring of neural activity both optically and electrically, and open further possibilities for a wide variety of studies to be explored in the future.

\section{Discussion}

The aim of this work was to demonstrate the feasibility to record optical and electrophysiological signals for the same site simultaneously in vivo. Previously, this has been problematic due to the highly reflective inorganic materials used to create electrodes and the photoelectric effect caused by the laser light beam at conduction lines and recording sites. We minimized these issues by making use of an organic array of electrodes on an optically transparent substrate. To accomplish this, a low impedance PEDOT:PSS-based microelectrode array was developed (Fig. 1) to ensure that a high level $(>95 \%)$ of the area of interest remains unobstructed for laser scanning, allowing for simultaneous fluorescence signal collection through 2P microscopy (Fig. 2). The mixed conduction PEDOT: PSS material system has received a great deal of attention due to its excellent electronic and mechanical properties, and the resulting practicality and commercial availability (Elschner et al., 2011). The material flexibility is an additional benefit, providing excellent contact with the cortical surface and reduced invasiveness compared to traditionally stiffer implantable devices (Khodagholy et al., 2015).

Over the past decades, neural interfacing systems using various types of transparent and flexible materials have increased in number (Stieglitz et al., 1997; Rodger et al., 2008; Ledochowitsch et al., 2011). To meet the needs of a wide-range of applications, there have been significant advances in graphene/carbon-based neural probes (David-Pur et al., 2013; Du et al., 2015) as well as those based on conjugated polymer systems (Mercanzini et al., 2008; Khodagholy et al., 2016). The advantages of PEDOT:PSS in neural interfacing studies have been previously demonstrated as well, showing improvement in recording quality while reducing tissue reactions (Khodagholy et al., 2011; Someya et al., 2016; Williamson et al., 2015). This mixed conduction material system has received a great deal of attention due to its excellent electronic and mechanical properties, and the resulting practicality and commercial availability (Elschner et al., 2011).Additionally, as a result of the success of PEDOT: PSS, a great deal of research has gone into understanding this polymer system and optimizing its mechanical and electrical properties (Crispin et al., 2006; Martin et al., 2010; Proctor et al., 2016; Rivnay et al., 2016). The prog- ress made in this area to date is significant for the field of bioelectronics (Rivnay et al., 2014). Making use of the development and optimization of PEDOT:PSS, electrodes of very small size can be engineered, while retaining low electrochemical impedance and thus maintaining excellent neural recording ability. These properties were taken advantage of here to enable simultaneous optical and electrical characterization of neural networks.

In this study, we were able to show seizure events with electrophysiological recordings and the corresponding optical signals form many neurons (Fig. 3). Comparison was made between optical data that were recorded from the same location as the PEDOT:PSS electrode used to obtain the electrical recording, i.e., Figure $3 A, B$. The simultaneous electrophysiological and optical recording strategy could be employed in future applications to extrapolate seizure foci in pathophysiological networks and, using readily available transgenic animals, to identify the exact neuronal cell types behind the pathologic activity using imaging in vivo. Moreover, because of the high scalability of this approach, it will be useful to investigate healthy physiologic systems. The size and shape of the of the overall grid are extremely adjustable and may be easily altered depending on the targeted application. In addition, the density of electrode sites may be adapted to optimize recording from specific brain regions. For example, the design and implementation of a grid that could cover the entire visual cortex, or simply particular subregions, is straightforward. Whereas previous studies have used relatively large electrode surfaces (on the scale of 50 to several hundred micrometers; Kuzum et al., 2014; Lu et al., 2016), micro-fabricated PEDOT:PSS electrodes easily allow for reduced electrode size to the $10-\mu \mathrm{m}$ scale while keeping the impedance in the biologically relevant/ desired range. Furthermore, although the metal contact lines will become problematic at very high site densities as a result of optical path obstruction and the photoelectric effect, the possibility to stack conduction lines vertically using these materials (Donahue et al., 2017) may be employed to increase site density while retaining high transparency. In addition to the low impedance of PEDOT: PSS electrode sites, which maintains the recording capability for biological signals, these sites can also be used for electrical stimulation (Wilks et al., 2009; Williamson et al., 2015). It could therefore be conceivable to take the advantage of this option of the micro-grid and combine local stimulation with simultaneous $2 \mathrm{P}$ imaging to assess signal propagation in neuronal networks. Lastly, as PEDOT:PSS electrodes have demonstrated good biocompatibility (Berggren and Richter-Dahlfors, 2007) as well as the ability to retain recording capability from weeks up to multiple months in vivo (Khodagholy et al., 2015; Kozai et al., 2016), this type of probe could also be employed for chronic implantation.

In summary, the present work demonstrates a novel method to perform classical electrophysiology recordings using a state-of-the-art microelectrode array along with $2 \mathrm{P}$ imaging of network activity in vivo. Initial results demonstrate good correlation of induced pathologic activity using this dual characterization method, providing the 
best of the two methods: high spatial resolution from $2 \mathrm{P}$ imaging combined with high temporal resolution of the multielectrode array. This approach may be developed further to include depth electrodes to simultaneously capture electrophysiological and $2 \mathrm{P}$ measurements of singlecell activity across networks.

\section{References}

Avoli M, D’Antuono M, Louvel J, Köhling R, Biagini G, Pumain R, D'Arcangelo G, Tancredi V (2002) Network and pharmacological mechanisms leading to epileptiform synchronization in the limbic system in vitro. Prog Neurobiol 68:167-207. CrossRef

Benfenati V, Toffanin S, Bonetti S, Turatti G, Pistone A, Chiappalone M, Sagnella A, Stefani A, Generali G, Ruani G, Saguatti D, Zamboni R, Muccini M (2013) A transparent organic transistor structure for bidirectional stimulation and recording of primary neurons. Nat Mater 12:672-680. CrossRef

Berggren M, Richter-Dahlfors A (2007) Organic bioelectronics. Adv Mater 19:3201-3213. CrossRef

Buzsáki G, Draguhn A (2004) Neuronal oscillations in cortical networks. Science 304:1926-1929. CrossRef Medline

Chang WP, Wu JS, Lee CM, Vogt BA, Shyu BC (2011) Spatiotemporal organization and thalamic modulation of seizures in the mouse medial thalamic-anterior cingulate slice. Epilepsia 52: 2344-2355. CrossRef

Crispin X, Jakobsson FLE, Crispin A, Grim PCM, Andersson P, Volodin A, van Haesendonck C, Van der Auweraer M, Salaneck WR, Berggren M (2006) The origin of the high conductivity of poly(3,4-ethylenedioxythiophene) - poly(styrenesulfonate) (PEDOTPSS) plastic electrodes. Chem Mater 18:4354-4360. CrossRef

Dana H, Chen TW, Hu A, Shields BC, Guo C, Looger LL, Kim DS, Svoboda K (2014) Thy1-GCaMP6 transgenic mice for neuronal population imaging in vivo. PloS one. 9:e108697. CrossRef Medline

David-Pur M, Bareket-Keren L, Beit-Yaakov G, Raz-Prag D, Hanein Y (2013) All-carbon-nanotube flexible multi-electrode array for neuronal recording and stimulation. Biomed Microdevices 16:4353. CrossRef

Donahue MJ, Williamson A, Strakosas X, Friedlein JT, McLeod RR, Gleskova H, Malliaras GG (2017) High-Performance Vertical Organic Electrochemical Transistors. Adv Mater 30:1705031. CrossRef

Du X, Wu L, Cheng J, Huang S, Cai Q, Jin Q, Zhao J (2015) Graphene microelectrode arrays for neural activity detection. J Biol Phys 41:339-347. CrossRef

Elschner A, Kirchmeyer S, Lovenich W, Merker U, Reuter K (2011) PEDOT: principles and applications of an intrinsically conductive polymer. Boca Raton, FL: CRC Press.

Franks W, Schenker I, Schmutz P, Hierlemann A (2005) Impedance characterization and modeling of electrodes for biomedical applications. IEEE Trans Biomed Eng 52:1295-1302. CrossRef

Helmchen F, Fee MS, Tank DW, Denk W (2001) A miniature headmounted two-photon microscope. high-resolution brain imaging in freely moving animals. Neuron 31:903-912. CrossRef

Katona G, Szalay G, Maák P, Kaszás A, Veress M, Hillier D, Chiovini B, Vizi ES, Roska B, Rózsa B (2012) Fast two-photon in vivo imaging with three-dimensional random-access scanning in large tissue volumes. Nat Methods 9:201-208. CrossRef

Khodagholy D, Doublet T, Gurfinkel M, Quilichini P, Ismailova E, Leleux P, Herve T, Sanaur S, Bernard C, Malliaras GG (2011) Highly conformable conducting polymer electrodes for in vivo recordings. Adv Mater 23:268-272. CrossRef Medline

Khodagholy D, Doublet T, Quilichini P, Gurfinkel M, Leleux P, Ghestem A, Ghestem A, Ismailova E, Hervé T, Sanaur S, Bernard C, Malliaras GG (2013) In vivo recordings of brain activity using organic transistors. Nat Commun 4:1575. CrossRef Medline
Khodagholy D, Gelinas JN, Thesen T, Doyle W, Devinsky O, Malliaras GG, Buzsáki G (2015) NeuroGrid: recording action potentials from the surface of the brain. Nat Neurosci 18:310-315. CrossRef

Khodagholy D, Gelinas JN, Zhao Z, Yeh M, Long M, Greenlee JD, Doyle W, Devinsky O, Buzsáki G (2016) Organic electronics for high-resolution electrocorticography of the human brain. Sci Adv 2:e1601027. CrossRef

Kitamura K, Judkewitz B, Kano M, Denk W, Häusser M (2008) Targeted patch-clamp recordings and single-cell electroporation of unlabeled neurons in vivo. Nat Methods 5:61-67. CrossRef

Kozai TDY, Vazquez AL (2015) Photoelectric artefact from optogenetics and imaging on microelectrodes and bioelectronics: new challenges and opportunities. J Mater Chem B 3:4965-4978. CrossRef

Kozai TDY, Catt K, Du Z, Na K, Srivannavit O, Haque R-uM, Seymour J, Wise KD, Yoon E, Cui XT (2016) Chronic in vivo evaluation of PEDOT/CNT for stable neural recordings. IEEE Trans Biomed Eng 63:111-119. CrossRef

Kuzum D, Takano H, Shim E, Reed JC, Juul H, Richardson AG, De Vries J, Bink H, Dichter MA, Lucas TH, Coulter DA, Cubukcu E, Litt B (2014) Transparent and flexible low noise graphene electrodes for simultaneous electrophysiology and neuroimaging. Nat Commun 5:5259. CrossRef Medline

Ledochowitsch P, Olivero E, Blanche T, Maharbiz MM (2011) A transparent $\mu \mathrm{ECoG}$ array for simultaneous recording and optogenetic stimulation. Conf Proc IEEE Eng Med Biol Soc 2011:29372940. CrossRef Medline

Lu Y, Lyu H, Richardson AG, Lucas TH, Kuzum D (2016) Flexible neural electrode array based-on porous graphene for cortical microstimulation and sensing. Sci Rep 6. 33526. CrossRef

Malvache A, Reichinnek S, Villette V, Haimerl C, Cossart R (2016) Awake hippocampal reactivations project onto orthogonal neuronal assemblies. Science 353:1280-1283. CrossRef Medline

Martin DC, Wu J, Shaw CM, King Z, Spanninga SA, RichardsonBurns S, Hendricks J, Yang J (2010) The morphology of poly(3,4ethylenedioxythiophene). Polym Rev 50:340-384. CrossRef

Mercanzini A, Cheung K, Buhl DL, Boers M, Maillard A, Colin P, Bensadoun JC, Bertsch A, Renaud P (2008) Demonstration of cortical recording using novel flexible polymer neural probes. Sens Actuators A Phys 143:90-96. CrossRef

Park DW, Schendel AA, Mikael S, Brodnick SK, Richner TJ, Ness JP, Hayat MR, Atry F, Frye ST, Pashaie R, Thongpang S, Ma Z, Williams JC (2014) Graphene-based carbon-layered electrode array technology for neural imaging and optogenetic applications. Nat Commun 5:5258. CrossRef Medline

Proctor CM, Rivnay J, Malliaras GG (2016) Understanding volumetric capacitance in conducting polymers. J Polym Sci B Polym Phys 54:1433-1436. CrossRef

Qiang Y, Artoni P, Seo KJ, Culaclii S, Hogan V, Zhao X, Zhong Y, Han $X$, Wang PM, Lo YK, Li Y, Patel HA, Huang Y, Sambangi A, Chu JSV, Liu W, Fagiolini M, Fang H (2018) Transparent arrays of bilayer-nanomesh microelectrodes for simultaneous electrophysiology and two-photon imaging in the brain. Sci Adv 4:eaat0626. CrossRef Medline

Rivnay J, Owens RM, Malliaras GG (2014) The rise of organic bioelectronics. Chem Mater 26:679-685. CrossRef

Rivnay J, Inal S, Collins BA, Sessolo M, Stavrinidou E, Strakosas X, Tassone C, Delongchamp DM, Malliaras GG (2016) Structural control of mixed ionic and electronic transport in conducting polymers. Nat Commun 7:11287. CrossRef

Rodger DC, Fong AJ, Li W, Ameri H, Ahuja AK, Gutierrez C, Lavrov I, Zhong H, Menon PR, Meng E, Burdick JW, Roy RR, Edgerton VR, Weiland JD, Humayun MS, Tai YC (2008) Flexible parylenebased multielectrode array technology for high-density neural stimulation and recording. Sens Actuators B Chem 132:449-460. CrossRef

Scott A, Weir K, Easton C, Huynh W, Moody WJ, Folch A (2013) A microfluidic microelectrode array for simultaneous electrophysiology, chemical stimulation, and imaging of brain slices. Lab Chip 13:527-535. CrossRef 
Sessolo M, Khodagholy D, Rivnay J, Maddalena F, Gleyzes M, Steidl E, Buisson B, Malliaras GG (2013) Easy-to-fabricate conducting polymer microelectrode arrays. Adv Mater 25:2135-2139. CrossRef

Someya T, Bao Z, Malliaras GG (2016) The rise of plastic bioelectronics. Nature 540:379-385. CrossRef

Stieglitz T, Beutel H, Meyer JU (1997) A flexible, light-weight multichannel sieve electrode with integrated cables for interfacing regenerating peripheral nerves. Sens Actuators A Phys 60:240-243. CrossRef

Stosiek C, Garaschuk O, Holthoff K, Konnerth A (2003) In vivo two-photon calcium imaging of neuronal networks. Proc Natl Acad Sci USA 100:7319-7324. CrossRef

Svoboda K, Yasuda R (2006) Principles of two-photon excitation microscopy and its applications to neuroscience. Neuron 50:823839. CrossRef

Szente M, Baranyi A (1987) Mechanism of aminopyridine-induced ictal seizure activity in the cat neocortex. Brain Res 413:368-373. CrossRef

Taketani M, Baudry M (2010) Advances in network electrophysiology. New York, NY: Springer.
Thunemann M, Lu Y, Liu X, Kılıç K, Desjardins M, Vandenberghe M, Sadegh S, Saisan PA, Cheng Q, Weldy KL, Lyu H, Djurovic S, Andreassen OA, Dale AM, Devor A, Kuzum D (2018) Deep 2-photon imaging and artifact-free optogenetics through transparent graphene microelectrode arrays. Nat Commun 9:2035. CrossRef Medline

Voskuyl RA, Albus H (1985) Spontaneous epileptiform discharges in hippocampal slices induced by 4 -aminopyridine. Brain Res 342: 54-66. CrossRef

Wilks SJ, Richardson-Burns SM, Hendricks JL, Martin DC, Otto KJ (2009) Poly(3,4-ethylenedioxythiophene) as a micro-neural interface material for electrostimulation. Front Neuroeng 2:7. CrossRef Medline

Williams JC, Hippensteel JA, Dilgen J, Shain W, Kipke DR (2007) Complex impedance spectroscopy for monitoring tissue responses to inserted neural implants. J Neural Eng 4:410. CrossRef

Williamson A, Ferro M, Leleux P, Ismailova E, Kaszas A, Doublet T, Quilichini P, Rivnay J, Rózsa B, Katona G, Bernard C, Malliaras GG (2015) Localized neuron stimulation with organic electrochemical transistors on delaminating depth probes. Adv Mater 27:44054410. CrossRef 